Research Article

\title{
Finding Damage Localizations of a Planar Truss Using Modal Strain Energy Change
}

\author{
Xiaoshun Wu $\left(\mathbb{D},{ }^{1}\right.$ Juwei Xia $\left(\mathbb{D},{ }^{2}\right.$ and Xuekun $\mathrm{Zhu}^{3}$ \\ ${ }^{1}$ Nanchang Campus, Jiangxi University of Science and Technology, Ganzhou, Jiangxi 341000, China \\ ${ }^{2}$ ZheJiang WuZhou Project Management Co., Ltd., Hangzhou, Zhejiang 310053, China \\ ${ }^{3}$ Department of Road \& Bridge Engineering, Jiangxi V\&T College of Communication, Nanchang, Jiangxi 330013, China
}

Correspondence should be addressed to Xiaoshun Wu; wuxiaoshun1981@163.com

Received 23 May 2019; Revised 12 August 2019; Accepted 28 August 2019; Published 17 September 2019

Academic Editor: Claudio Mazzotti

Copyright (C) 2019 Xiaoshun Wu et al. This is an open access article distributed under the Creative Commons Attribution License, which permits unrestricted use, distribution, and reproduction in any medium, provided the original work is properly cited.

\begin{abstract}
A novel structural damage localization method based on modal strain energy changes is proposed. The proposed method does not utilize the first several modes together as usual but selectively makes use of some sensitive modes for each element. Both the magnitude of normalized elemental modal strain energy and the capability of each element to resist identification uncertainties are considered to determine the sensitive modes. Elements' capability to resist identification uncertainties is calculated by using the Monte Carlo method. Based on determined sensitive modes, an in-turn damage-checking process is employed to judge the damage state of each element. A typical planar truss is numerically analyzed. The results show that the proposed method is more effective than that based on modal strain energy change ratio even when using the first several modes together as usual. Furthermore, by utilizing some sensitive modes only, the proposed method becomes much more robust, making damage localization more easy and reliable.
\end{abstract}

\section{Introduction}

Civil infrastructures such as buildings, bridges, towers, and offshore platforms have been widely constructed in our highly industrialized society. They are designed to serve for a long time, but many factors (like environmental corrosion and extreme loadings) can cause structural damage and reduce their lifetime. Structural health monitoring $[1,2]$ is thus required to guarantee the lifetime safety of the infrastructures. As an efficient tool to evaluate the health of a structure, many vibration-based damage detection methods [3-5] have been developed to identify structural damage in a nondestructive way. These methods utilize the changes in modal parameters caused by local damage, including the changes of modal eigenvalues, mode shapes, and mode shape curvatures [6]. The changes of modal parameters' combinations like modal flexibility [7], modal residual force [8], modal strain energy [9], etc., are also broadly used in the field of vibration-based damage detection.

Special attention is paid on the modal strain energy in this paper, which has been extensively employed in locating structural damages [9-14]. Shi et al. [9] presented a method using the change ratio of elemental modal strain energy between the intact and damaged structures, theoretically and numerically finding that the modal strain energy change of a damaged element is usually larger than that of any other intact elements. Yan et al. [10] presented a statistic structural damage detection algorithm using the closed form of elemental modal strain energy sensitivity to consider the uncertainties existing in the structural model and measured structural modal parameters. Seyedpoor [11] proposed a two-stage method to identify the location and severity of multiple structural damage using a modal strain energybased index at the first stage. Vo-Duy et al. [12] proposed a two-step approach to detect damages in laminated composite structures, which utilizes the same indicator presented in [9] to locate damages at the first step. Tan et al. [13] detected damages in steel beams utilizing modal strain energy-based damage index and Artificial Neural Network, considering only the first vibration mode. Liu et al. [14] proposed a method to detect structural damages of offshore platforms by introducing the concept of grouping modal 
strain energy, which divided the elemental modal strain energy into axial tension-compression and bending. By employing optimization algorithms $[11,12]$ or formulating sensitivity matrices $[15,16]$, modal strain energy can be further used to quantify structural damage. According to the studies, the modal strain energy-based damage indicators are sensitive to local damage.

In a sense, damage localization plays a more important role than damage quantification. If damaged elements are correctly located, the damage quantification accuracy and computation efficiency will correspondingly be improved [8]. Thus, damage localization is focused on in this paper. It is noticed that current studies normally used the first several modes together to calculate damage indicators to ensure their robustness. However, Doebling et al. [17] found that the mode storing maximum modal strain energy (may not be the first mode) could produce more accurate damage detections than the first mode, meaning that the sensitivity of modes to structural damage is different. How to find sensitive modes to improve damage detection accuracy is still an interesting topic.

In this paper, a new damage localization method is proposed, which establishes a new damage location indicator and uses properly selected sensitive modes. Firstly, the new damage location indicator is proposed by using normalized modal strain energy changes before and after damage. Secondly, the concept of sensitive modes is presented. The criteria of selecting sensitive modes, which are related to the elemental modal strain energy and the elemental resistance ability to identification uncertainties, are discussed. Finally, a simply supported planar truss is numerically analyzed to illustrate the damage detection process and verify the effectiveness of the proposed method.

\section{Modal Strain Energy-Based Damage Indicators}

The eigenvalue equation of an undamped intact structure is written as

$$
\left(\mathbf{K}-\lambda_{i} \mathbf{M}\right) \boldsymbol{\theta}_{i}=\mathbf{0},
$$

where $\mathbf{K}$ is the global structural stiffness matrix; $\mathbf{M}$ is the consistent mass matrix; $\lambda_{i}$ is the eigenvalue of the $i$-th mode; and $\boldsymbol{\theta}_{i}$ is the mass-normalized mode shape vector of the $i$-th mode. Thus, $\boldsymbol{\theta}_{i}^{\mathrm{T}} \mathbf{K} \boldsymbol{\theta}_{i}=\lambda_{i}$, and $\boldsymbol{\theta}_{i}^{\mathrm{T}} \mathbf{M} \boldsymbol{\theta}_{i}=1$.

Likewise, the eigenvalue equation of the damaged structure is described as

$$
\left(\mathbf{K}^{\mathrm{d}}-\lambda_{i}^{\mathrm{d}} \mathbf{M}^{\mathrm{d}}\right) \boldsymbol{\theta}_{i}^{\mathrm{d}}=\mathbf{0},
$$

where the superscript $d$ refers to the damaged structure. It is assumed that the mass of a structure can be well estimated and has no loss before and after damage. Meanwhile, only a loss of stiffness in one or more elements takes place when structural damage occurs. The stiffness matrix of the $j$-th element of the damaged structure is written as

$$
\mathbf{K}_{j}^{\mathrm{d}}=\left(1-\alpha_{j}\right) \mathbf{K}_{j},
$$

where $\mathbf{K}_{j}$ is the stiffness matrix of the $j$-th element of the intact structure and $\alpha_{j}$ is the damage extent of the $j$-th element, and $0 \leq \alpha_{j}<1$. Because $\mathbf{K}=\sum_{j}^{b} \mathbf{K}_{j}$, the global stiffness matrix of the damaged structure can then be expressed by

$$
\mathbf{K}^{\mathrm{d}}=\mathbf{K}-\sum_{j}^{b} \alpha_{j} \mathbf{K}_{j}
$$

where $b$ is the total number of elements.

For the intact structure, the modal strain energy (MSE) of the $j$-th element at the $i$-th mode is defined as [9]

$$
\mathrm{MSE}_{i j}=\boldsymbol{\theta}_{i}^{\mathrm{T}} \mathbf{K}_{j} \boldsymbol{\theta}_{i},
$$

where the superscript T refers to the transpose of a vector or matrix. Correspondingly, the MSE of the $j$-th element at the $i$-th mode is expressed as

$$
\operatorname{MSE}_{i j}^{\mathrm{d}}=\left(\boldsymbol{\theta}_{i}^{\mathrm{d}}\right)^{\mathrm{T}} \mathbf{K}_{j} \boldsymbol{\theta}_{i}^{\mathrm{d}} .
$$

It is noted that $\mathbf{K}_{j}$ instead of $\mathbf{K}_{j}^{\mathrm{d}}$ is utilized in calculating $\mathrm{MSE}_{i j}^{\mathrm{d}}$ because the true damage extent of the $j$-th element is unknown.

The modal strain energy change (MSEC) of the $j$-th element at the $i$-th mode before and after structural damage could then be obtained by

$$
\mathrm{MSEC}_{i j}=\mathrm{MSE}_{i j}^{\mathrm{d}}-\mathrm{MSE}_{i j} \text {. }
$$

Consequently, the modal strain energy change ratio (MSECR) is defined as

$$
\operatorname{MSECR}_{i j}=\frac{\left|\mathrm{MSE}_{i j}^{\mathrm{d}}-\mathrm{MSE}_{i j}\right|}{\mathrm{MSE}_{i j}} .
$$

Usually, the first several modes are considered together to compute the averaged MSECR which is described by [9]

$$
\operatorname{MSECR}_{j}=\sum_{i=1}^{m} \frac{\operatorname{MSECR}_{i j}}{\operatorname{MSECR}_{i, \max }},
$$

where $m$ is the number of the selected modes. MSECR ${ }_{i \text {,max }}$ takes the form

$$
\operatorname{MSECR}_{i, \max }=\max _{k}\left(\operatorname{MSECR}_{i k}\right), \quad k=1,2,3, \ldots, b,
$$

where $\max (\cdot)$ is the function to take the maximum. A large $\mathrm{MSECR}_{j}$ may indicate a damaged element $j$. The classic MSECR has been validated as a good indicator to find structural damage sites. However, sometimes it is not robust enough and even loses effectiveness when considering the influence of identification uncertainties.

A novel indicator called modal strain energy change index (MSECI) is thus put forward with an expression as

$$
\operatorname{MSECI}_{j}=\max \left(0, \frac{1}{m} \sum_{i=1}^{m} \frac{\mathrm{MSEC}_{i j}}{\mathrm{MSEC}_{i, \max }}\right) \text {, }
$$

where $\mathrm{MSEC}_{i, \max }=\max \left(\mathrm{MSEC}_{i k}\right), k=1,2,3, \ldots, b$. It is noticed that $\mathrm{MSECI}_{j}$ is nonnegative and a large $\mathrm{MSECI}_{j}$ may indicate a damaged element $j$. The MSECI is actually the average of normalized MSEC considering several modes. 


\section{Selection of Sensitive Modes for the MSECI Method}

Although the classic MSECR is used very often [9, 12], it sometimes loses effectiveness even considering the first several modes together. The reason is that the MSECR of an undamaged element with small MSE (the denominator in equation (8)) but relatively large MSEC (the numerator in equation (8)) could be much greater than that of a damaged element with large MSE but relatively small MSEC. The proposed MSECI thus could perform better because the MSEC of a damaged element is normally larger than that of any other undamaged element [9], although there occasionally exist some exceptions. Therefore, the following discussion is merely focused on the proposed MSECI.

Intuitively speaking, the contributions of different modes to the MSEC of each element must be different. The modes that have great contributions to the MSEC of an element are defined as the sensitive modes of the element. Two factors are considered to measure modes' contributions for properly selecting sensitive modes as illustrated as follows.

\subsection{Selection of Potential Sensitive Modes Based on Elemental} MSE. The magnitude of normalized elemental MSE of the intact structure is the first factor. For a specific mode, the MSE of each element is calculated and then normalized with respect to the largest value. A threshold $\gamma_{\mathrm{MSE}}$ is prescribed (e.g., 0.10). If the normalized MSE of an element is greater than $\gamma_{\mathrm{MSE}}$, the mode is regarded as a potential sensitive mode of the element. In this way, some potential sensitive modes can be selected from the first several modes that can be easily measured. This principle of selecting potential sensitive modes cannot be strictly proved, but can be qualitatively explained as follows. For each mode, the smaller the normalized MSE of an element, the closer to structural nodal points the element could be. Hence, if a damaged element has very small normalized MSE, it may also have a small MSEC, but other undamaged elements far from nodal points may have relatively greater MSEC [9]. As a result, too small normalized MSE of an element may be unfavorable for indicating the damaged element when using the proposed MSECI. Thus, only the modes which produce great enough normalized MSE are selected as potential sensitive modes.

3.2. Selection of Potential Sensitive Modes considering Identification Uncertainties. The capability of elements to resist identification uncertainties (i.e., the sensitivity of elements to identification uncertainties) is the second factor. Identification uncertainties can be commonly observed in dynamic testing. It is assumed that mode shapes have been identified and normalized by the mass matrix for both intact and damaged structures. In order to consider the effect of identification uncertainties, polluted mode shapes of the damaged structure are given by [16].

$$
\boldsymbol{\theta}_{i k}^{\mathrm{d}}=\boldsymbol{\theta}_{i k}^{\mathrm{d}^{*}}(1+\varepsilon \cdot \text { rand }),
$$

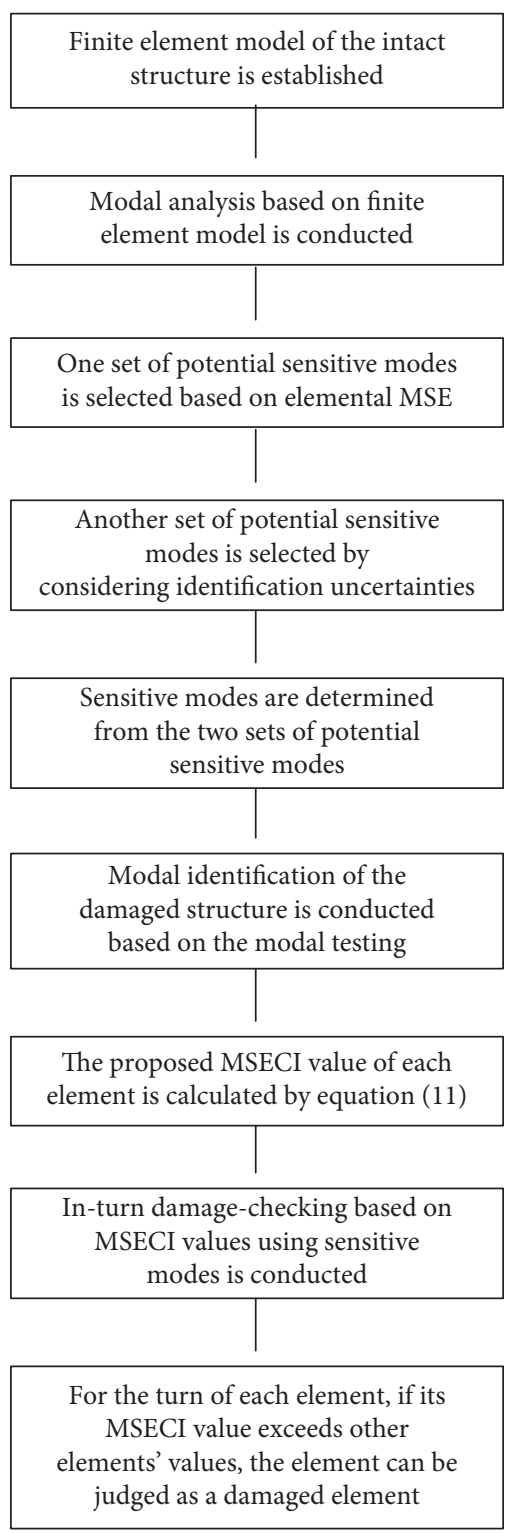

Figure 1: The flow chart of the proposed MSECI method.

where $\boldsymbol{\theta}_{i k}^{\mathrm{d}}$ and $\boldsymbol{\theta}_{i k}^{\mathrm{d}^{*}}$ are the polluted and unpolluted values of the $i$-th mode shape at the $k$-th degree of freedom, respectively; $\varepsilon$ is the noise level; rand is a random value between $[-1,1]$, yielding the uniform distribution. Because identification uncertainties can mathematically be regarded as a kind of noise, sometimes "noise" is still used instead of "identification uncertainties" in the subsequent paragraphs.

Correspondingly, the MSEC of the $j$-th element at the $i$-th mode polluted by noise can be given by

$$
\operatorname{MSEC}_{i j}=\mathrm{MSEC}_{i j}^{*}+\operatorname{MSEC}_{i j}^{N} \text {, }
$$

where $\mathrm{MSEC}_{i j}^{*}$ refers to the unpolluted MSEC of the $j$-th element at the $i$-th mode; MSEC $_{i j}^{N}$ is the error term caused by the identification uncertainties of mode shapes.

In order to measure the capability of an element to resist noise for computing its MSEC, the relative fluctuation 


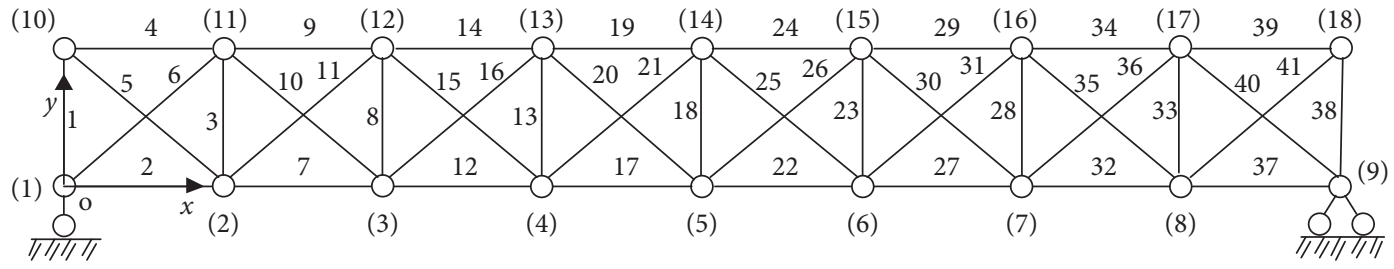

FIgURE 2: A planar truss with 41 elements (figures within brackets are the node number, and other figures are element number).

TABLE 1: First ten modal frequencies of the intact structure.

\begin{tabular}{lcccccccccc}
\hline Mode & 1 & 2 & 3 & 4 & 5 & 6 & 7 & 8 & 9 & 10 \\
\hline Frequency $(\mathrm{Hz})$ & 16.89 & 57.62 & 78.47 & 137.09 & 207.53 & 233.78 & 317.62 & 364.69 & 415.67 & 496.55 \\
\hline
\end{tabular}

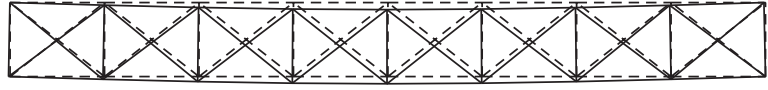

(a)

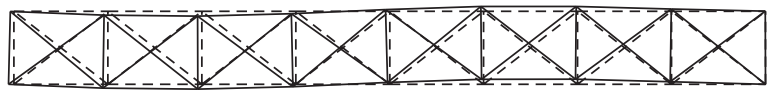

(c)

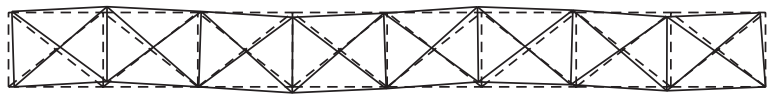

(e)

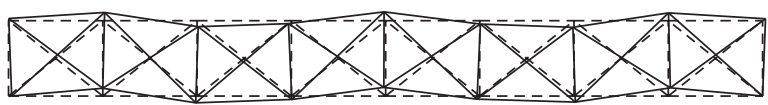

(g)

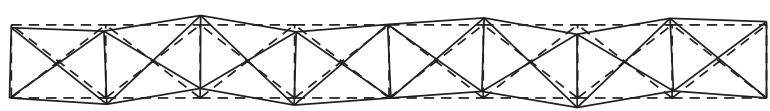

(i)

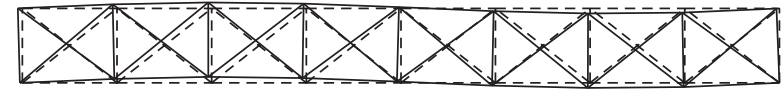

(b)

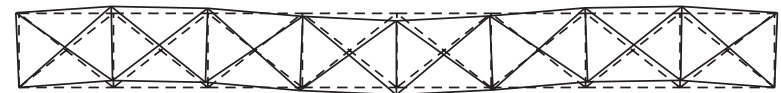

(d)

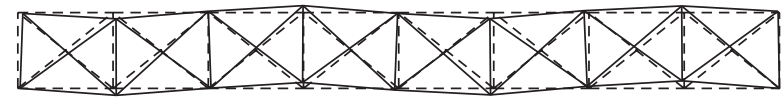

(f)

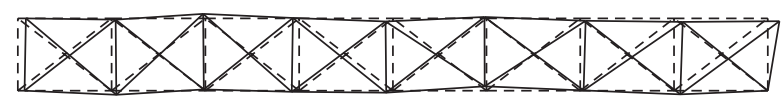

(h)

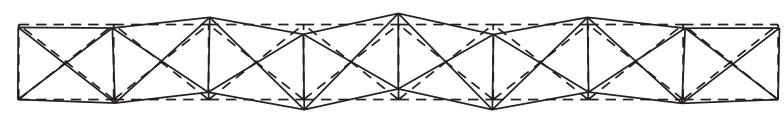

(j)

Figure 3: Mode shapes of the first ten modes of the planar truss (the thin dashed lines represent the equilibrium configuration, while the thick solid lines denote modal displacements enlarged by 2 times): (a) mode 1, (b) mode 2, (c) mode 3, (d) mode 4, (e) mode 5, (f) mode 6, (g) mode 7, (h) mode 8, (i) mode 9, and (j) mode 10.

amplitude (RFA) of the MSEC of the $j$-th element at the $i$-th mode based on a statistic analysis is defined as

$$
\operatorname{RFA}_{i j}=\max \left(\frac{\operatorname{MSEC}_{i j}^{N}}{\operatorname{MSEC}_{i j}^{*}}\right)-\min \left(\frac{\operatorname{MSEC}_{i j}^{N}}{\operatorname{MSEC}_{i j}^{*}}\right)
$$

where min $(\cdot)$ means taking the minimum value from all samples. $\mathrm{RFA}_{i j}$ is actually a variation range. The larger the $\mathrm{RFA}_{i j}$, the greater the influence of noise on the MSEC $i j$. $\mathrm{RFA}_{i j}$ is mainly affected by the factors including damage scenarios and noise levels. To simplify the calculation, it is assumed that the only element $j$ itself is damaged when computing $\mathrm{RFA}_{i j}$. In this paper, a 30\% damage extent and a $5 \%$ noise level (by equation (12)) is considered.

A threshold $\gamma_{\mathrm{RPA}}$ is then prescribed (e.g., 2.00). For each mode, if the RPA of an element is smaller than $\gamma_{\mathrm{RPA}}$, the mode should be treated as a potential sensitive mode of the element.
3.3. Determination of Sensitive Modes from Potential Sensitive Modes. As discussed above, two assemblies of potential sensitive modes are determined for each element by considering the normalized elemental MSE in Section 3.1 and the capability of the element to resist identification uncertainties in Section 3.2, respectively. The mutual modes in the two assemblies of an element are finally selected as the element's sensitive modes.

It is worth mentioning that the number of sensitive modes of an element may equal to zero, meaning that the damage state of the element cannot be reliably indicated by the proposed MSECI method. Whether an element has sensitive modes or not depends on the configuration of the structure, like geometry, topology, etc. Each element usually has different sensitive modes, so an in-turn damage-checking process is employed, as illustrated in Section 4.5. The flow chart of the proposed MSECI method is drawn in Figure 1. 


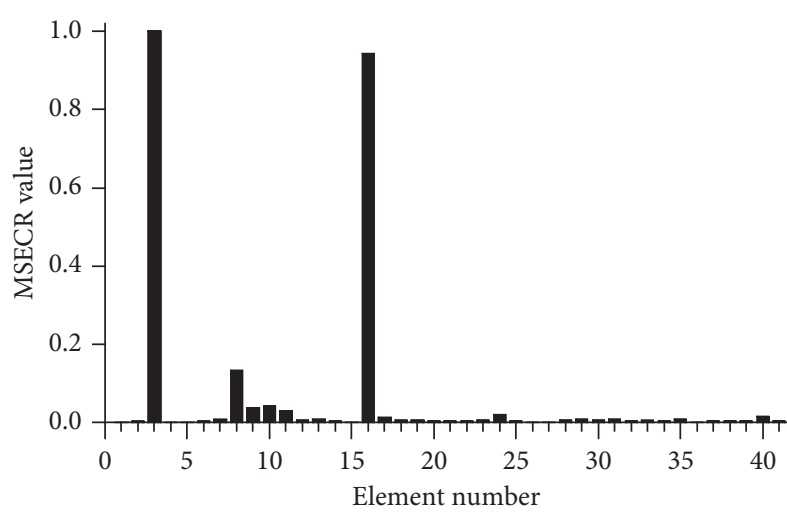

(a)

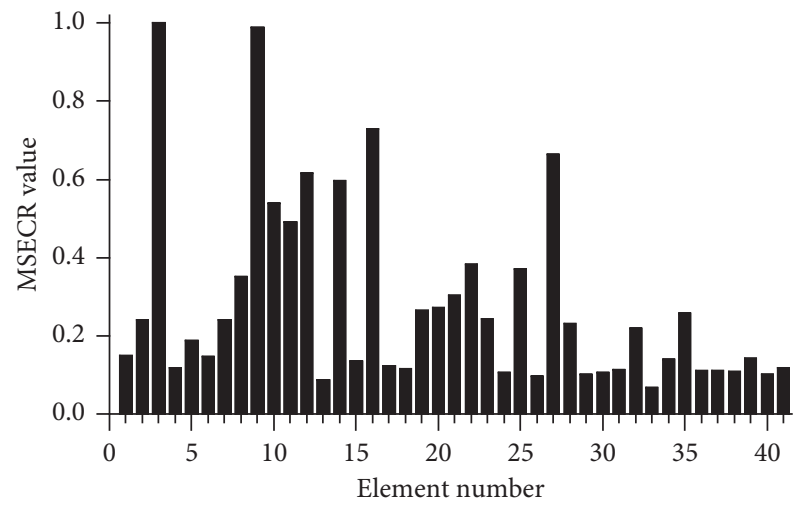

(c)

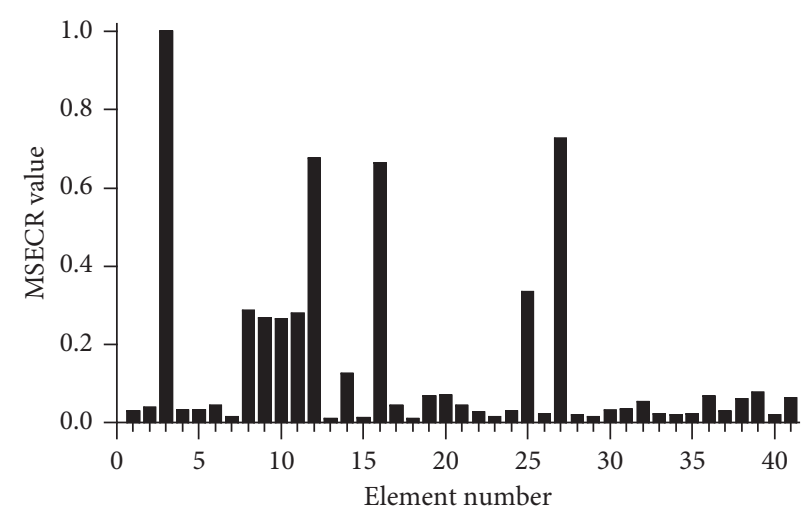

(b)

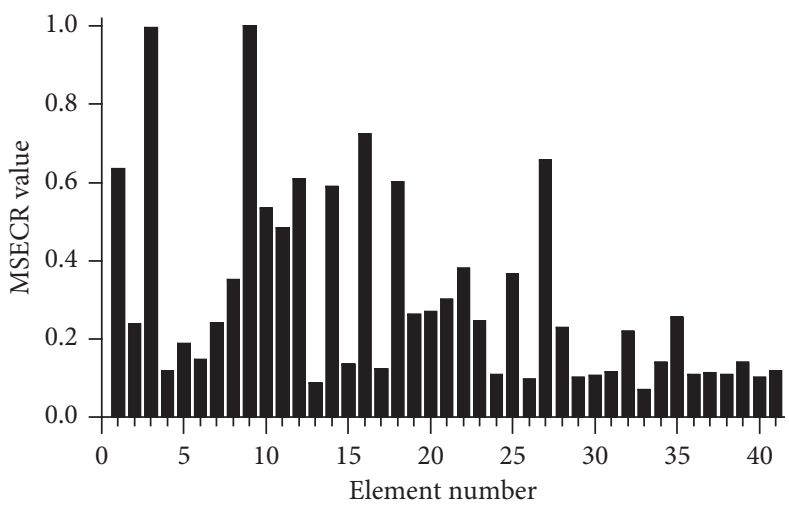

(d)

FIGURE 4: Values of the classic MSECR using the first several modes together (damaged element 9, without noise). (a) Using modes 1-2. (b) Using modes 1-4. (c) Using modes 1-6. (d) Using modes 1-8.

\section{Example and Verification}

4.1. Model Parameters. A simply supported planar truss is used to illustrate the process of selecting sensitive modes and to demonstrate the capability of the proposed MSECI method. The truss is shown in Figure 2, having 18 nodes and 41 elements. It is noted that there are no internal nodes at the intersections of web members. The truss is $1.2 \mathrm{~m}$ deep and $12 \mathrm{~m}$ long, with each upper or lower chord element having the same length of $1.5 \mathrm{~m}$. The cross-sectional area of each element is $0.001 \mathrm{~m}^{2}$. Young's Modulus and material density of each element are $206 \mathrm{GPa}$ and $7850 \mathrm{~kg} / \mathrm{m}^{3}$, respectively.

The structural damage is simulated by reducing Young's Modulus of damaged elements. Besides, when modal analysis for the truss is conducted, only the structural selfweight is considered. The first ten modal frequencies of the intact structure are shown in Table 1, while the mode shapes are shown in Figure 3.

4.2. Damage Localization Using the First Several Modes Together. The performances of the classic MSECR method by using equation (9) and the proposed MSECI method by using equation (11) are investigated using the first several modes together. It is assumed that the damage extent of element 9 is $30 \%$.
The MSECR values of all elements without noise are plotted in Figure 4. It reveals that even without noise, the MSECR method performs badly. According to Figure 4(a)4(b), when using the first two or four modes, the MSECR values of the damaged element are too small to indicate its damage state. From Figure 4(c)-4(d), although the damaged element has large MSECR values by using the first six or eight modes, serious disturbances from undamaged elements also appear. The MSECR values considering a random 5\% noise are depicted in Figure 5. Obviously, the MSECR method performs much worse in this case than in the case of free noise.

The proposed MSECI values considering free noise are drawn in Figure 6, in which the damaged element is clearly indicated. However, it is interesting that the disturbances from undamaged elements increase slightly with the growth of the number of used modes. The MSECI values with a random 5\% noise are plotted in Figure 7 . From Figure 7(a)-7(b), when using the first two or four modes, the damaged element can be located but with some other elements misjudged. From Figure $7(\mathrm{c})-7(\mathrm{~d})$, it becomes much easier to detect the damaged element by using the first six or eight modes. Generally speaking, the more modes are used, the more clearly the damaged element is indicated.

It is inferred from above analysis that the proposed MSECI method is more effective than the classic MSECR 


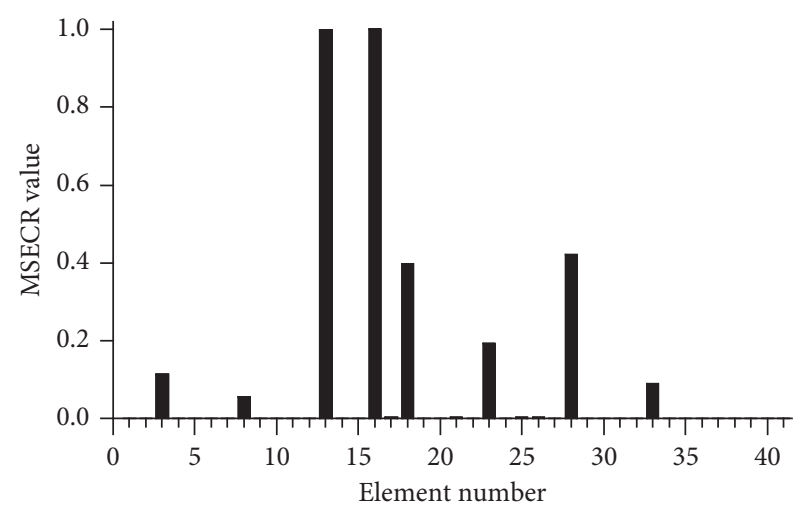

(a)

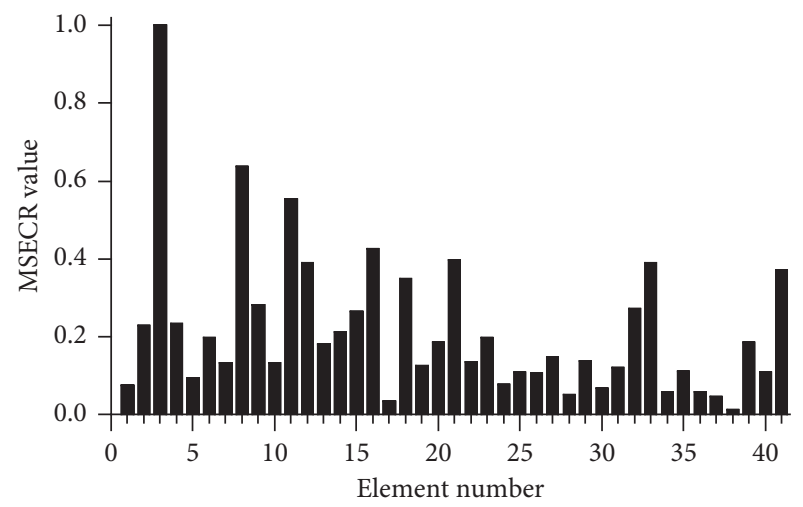

(c)

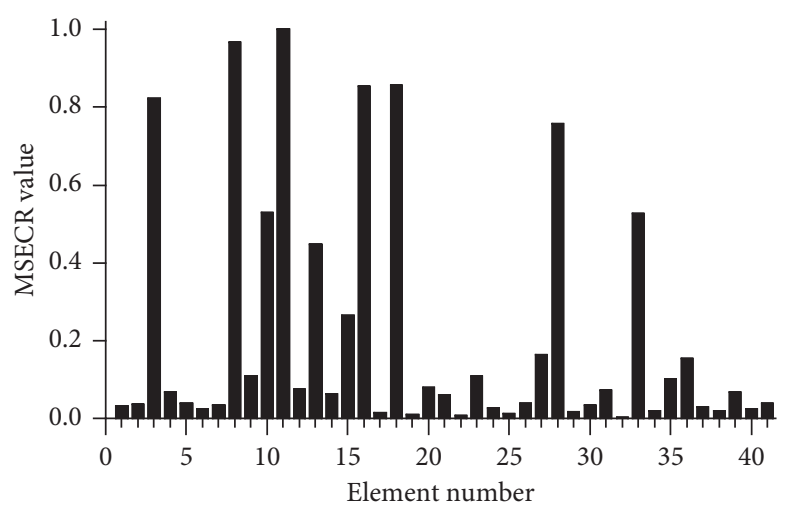

(b)

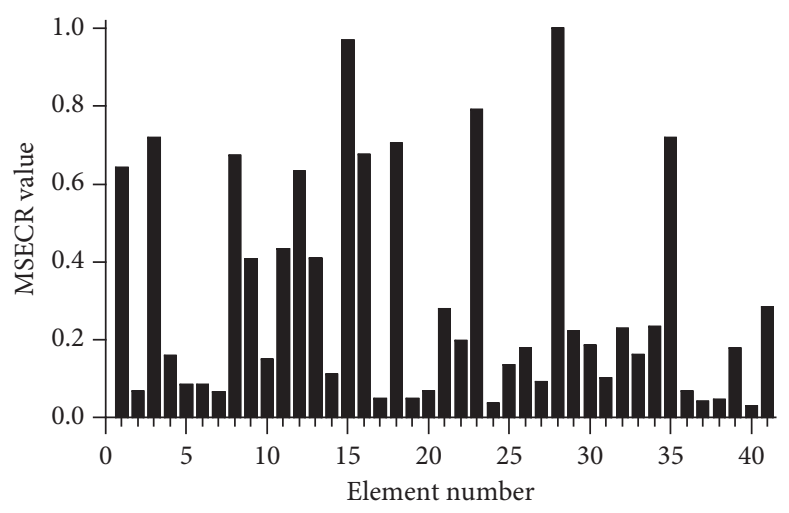

(d)

Figure 5: Values of the classic MSECR using the first several modes together (damaged element 9, with 5\% noise). (a) Using modes 1-2. (b) Using modes 1-4. (c) Using modes 1-6. (d) Using modes 1-8.

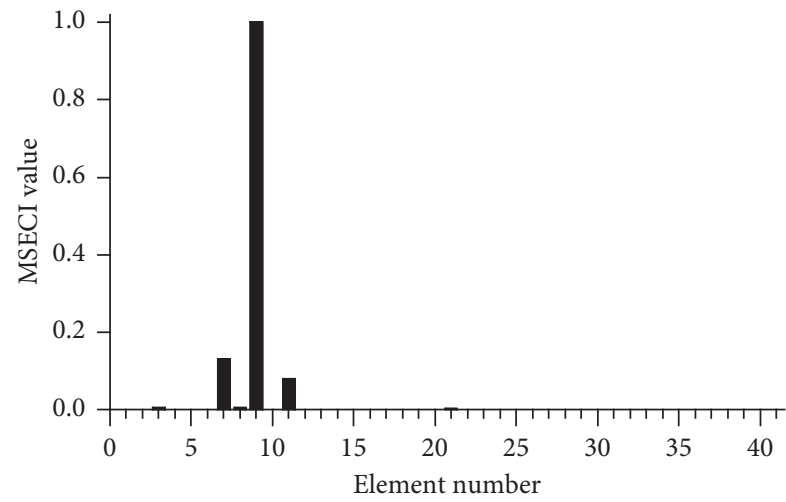

(a)

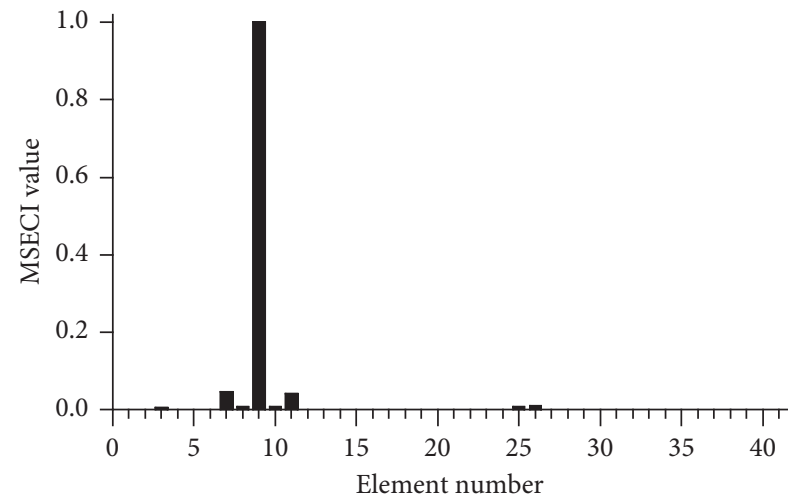

(b)

FIgURE 6: Continued. 


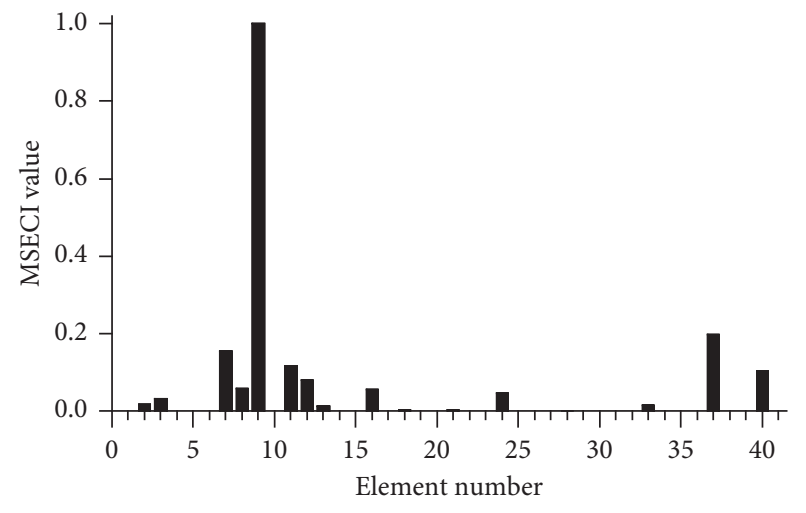

(c)

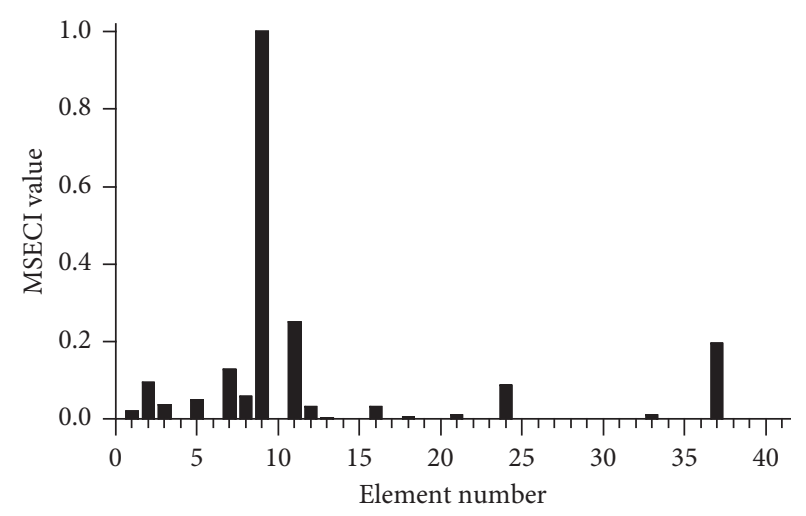

(d)

FIGURE 6: Values of the proposed MSECI using the first several modes together (damaged element 9, without noise). (a) Using modes 1-2. (b) Using modes 1-4. (c) Using modes 1-6. (d) Using modes 1-8.

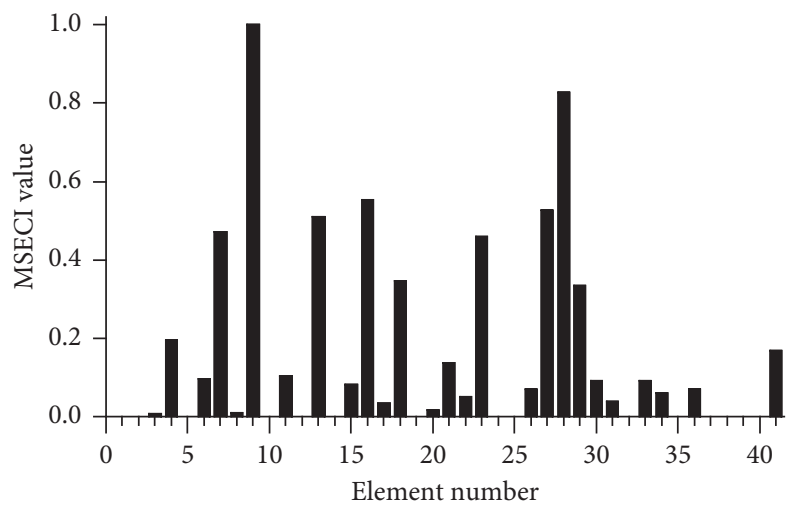

(a)

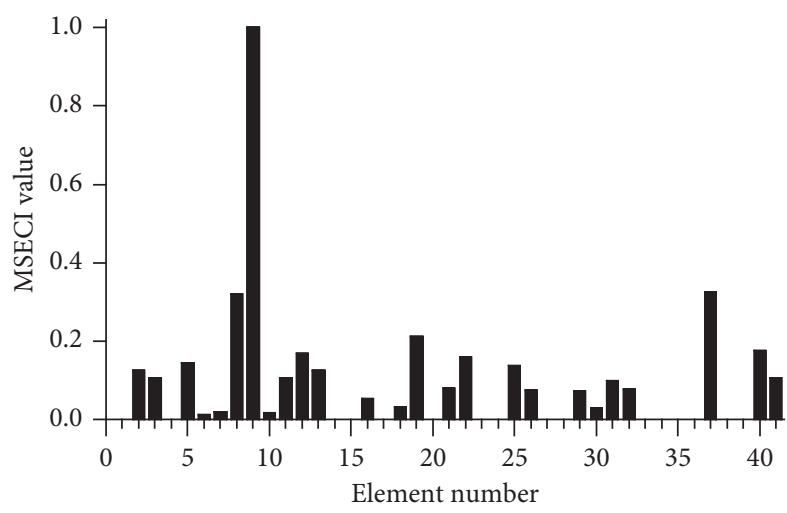

(c)

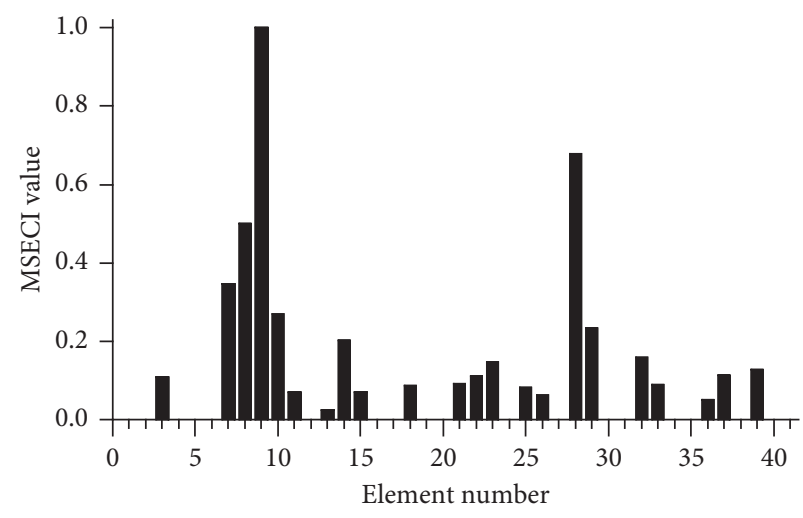

(b)

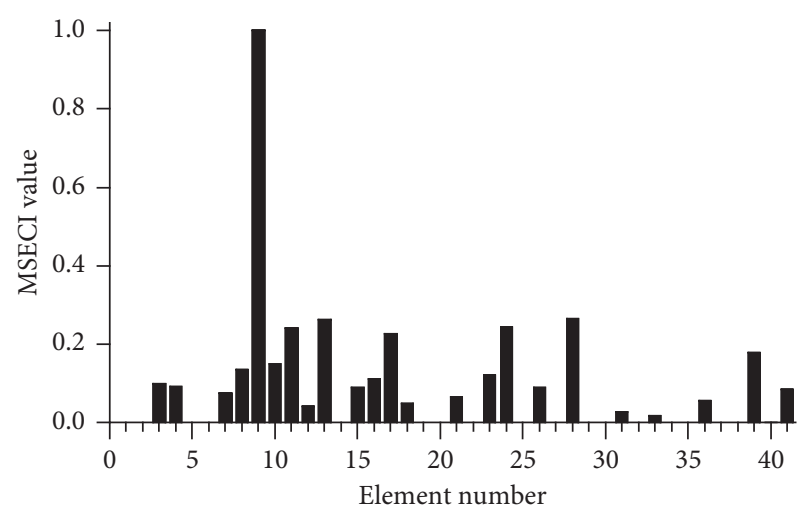

(d)

Figure 7: Values of the proposed MSECI using the first several modes together (damaged element 9, with 5\% noise). (a) Using modes 1-2. (b) Using modes 1-4. (c) Using modes 1-6. (d) Using modes 1-8.

method, at least in this example. Thus, the following discussion is associated only with the proposed MSECI method.

4.3. Selecting Sensitive Modes for the Proposed MSECI Method. Before investigating the performance of the proposed MSECI method, sensitive modes of all elements should be properly selected.
Firstly, potential sensitive modes are selected according to the principle suggested in Section 3.1. The normalized elemental MSE of the intact structure for the first ten modes are listed in Table 2. All normalized elemental MSE values that are larger than $\gamma_{\mathrm{MSE}}$ (prescribed as 0.10) are highlighted in bold. For each element, the mode having a highlighted value can be treated as a potential sensitive mode of the element. From Table 2, it reveals that when considering the 
TABLE 2: Normalized elemental MSE of the intact structure for the first ten modes.

\begin{tabular}{|c|c|c|c|c|c|c|c|c|c|c|}
\hline Element & Mode 1 & Mode 2 & Mode 3 & Mode 4 & Mode 5 & Mode 6 & Mode 7 & Mode 8 & Mode 9 & Mode 10 \\
\hline 1 & 0.010 & 0.012 & 0.021 & 0.074 & 0.053 & 0.037 & 0.186 & 0.000 & 0.223 & 0.121 \\
\hline 2 & 0.042 & 0.037 & 0.096 & 0.201 & 0.208 & 0.038 & 0.358 & 0.065 & 0.226 & 0.061 \\
\hline 3 & 0.000 & 0.001 & 0.002 & 0.003 & 0.016 & 0.017 & 0.004 & 0.119 & 0.000 & 0.000 \\
\hline 4 & 0.032 & 0.054 & 0.025 & 0.237 & 0.010 & 0.185 & 0.096 & 0.242 & 0.066 & 0.009 \\
\hline 5 & 0.057 & 0.057 & 0.096 & 0.256 & 0.147 & 0.064 & 0.246 & 0.009 & 0.133 & 0.035 \\
\hline 6 & 0.068 & 0.090 & 0.075 & 0.390 & 0.068 & 0.207 & 0.232 & 0.108 & 0.162 & 0.048 \\
\hline 7 & 0.306 & 0.191 & 0.494 & 0.474 & 0.477 & 0.004 & 0.081 & 0.607 & 0.018 & 0.037 \\
\hline 8 & 0.000 & 0.003 & 0.011 & 0.005 & 0.053 & 0.039 & 0.015 & 0.171 & 0.002 & 0.000 \\
\hline 9 & 0.291 & 0.371 & 0.131 & 0.872 & 0.008 & 0.371 & 0.023 & 0.584 & 0.108 & 0.080 \\
\hline 10 & 0.047 & 0.006 & 0.033 & 0.046 & 0.005 & 0.340 & 0.558 & 0.452 & 0.697 & 0.194 \\
\hline 11 & 0.043 & 0.031 & 0.000 & 0.006 & 0.263 & 0.043 & 1.000 & 0.034 & 0.912 & 0.251 \\
\hline 12 & 0.692 & 0.179 & 0.532 & 0.002 & 0.073 & 0.613 & 0.129 & 0.522 & 0.014 & 0.016 \\
\hline 13 & 0.000 & 0.006 & 0.023 & 0.010 & 0.071 & 0.045 & 0.007 & 0.035 & 0.000 & 0.001 \\
\hline 14 & 0.651 & 0.487 & 0.034 & 0.104 & 0.610 & 0.007 & 0.605 & 0.252 & 0.040 & 0.022 \\
\hline 15 & 0.023 & 0.025 & 0.001 & 0.599 & 0.003 & 0.304 & 0.107 & 0.041 & 1.000 & 0.648 \\
\hline 16 & 0.019 & 0.000 & 0.111 & 0.319 & 0.418 & 0.004 & 0.009 & 0.231 & 0.914 & 0.669 \\
\hline 17 & 0.976 & 0.013 & 0.184 & 0.882 & 0.034 & 0.410 & 0.169 & 0.012 & 0.133 & 0.013 \\
\hline 18 & 0.000 & 0.010 & 0.036 & 0.013 & 0.055 & 0.026 & 0.000 & 0.030 & 0.003 & 0.001 \\
\hline 19 & 0.910 & 0.239 & 0.090 & 0.235 & 0.771 & 0.021 & 0.061 & 0.018 & 0.228 & 0.000 \\
\hline 20 & 0.004 & 0.127 & 0.011 & 0.239 & 0.295 & 0.042 & 0.613 & 0.007 & 0.206 & 1.000 \\
\hline 21 & 0.002 & 0.021 & 0.268 & 0.054 & 0.003 & 0.418 & 0.477 & 0.010 & 0.153 & 0.881 \\
\hline 22 & 1.000 & 0.130 & 0.008 & 0.863 & 0.358 & 0.022 & 0.053 & 0.317 & 0.280 & 0.008 \\
\hline 23 & 0.000 & 0.014 & 0.050 & 0.013 & 0.021 & 0.003 & 0.007 & 0.168 & 0.003 & 0.000 \\
\hline 24 & 0.915 & 0.011 & 0.634 & 0.193 & 0.044 & 0.369 & 0.170 & 0.394 & 0.105 & 0.001 \\
\hline 25 & 0.001 & 0.183 & 0.001 & 0.065 & 0.369 & 0.024 & 0.633 & 0.006 & 0.187 & 0.904 \\
\hline 26 & 0.003 & 0.027 & 0.215 & 0.283 & 0.023 & 0.171 & 0.481 & 0.340 & 0.102 & 0.971 \\
\hline 27 & 0.753 & 0.625 & 0.001 & 0.003 & 0.272 & 0.065 & 0.614 & 0.403 & 0.018 & 0.026 \\
\hline 28 & 0.000 & 0.019 & 0.061 & 0.011 & 0.000 & 0.004 & 0.014 & 0.126 & 0.000 & 0.002 \\
\hline 29 & 0.665 & 0.058 & 0.971 & 0.174 & 0.029 & 0.060 & 0.128 & 0.842 & 0.060 & 0.009 \\
\hline 30 & 0.014 & 0.127 & 0.118 & 0.273 & 0.013 & 0.175 & 0.100 & 0.304 & 0.884 & 0.654 \\
\hline 31 & 0.022 & 0.002 & 0.052 & 0.621 & 0.096 & 0.181 & 0.006 & 0.097 & 1.000 & 0.709 \\
\hline 32 & 0.375 & 1.000 & 0.141 & 0.358 & 0.110 & 0.003 & 0.020 & 0.142 & 0.083 & 0.041 \\
\hline 33 & 0.000 & 0.024 & 0.079 & 0.010 & 0.014 & 0.036 & 0.013 & 0.000 & 0.009 & 0.000 \\
\hline 34 & 0.304 & 0.143 & 0.526 & 1.000 & 0.019 & 0.286 & 0.087 & 0.170 & 0.038 & 0.096 \\
\hline 35 & 0.035 & 0.040 & 0.415 & 0.000 & 0.285 & 0.001 & 0.567 & 0.023 & 0.748 & 0.239 \\
\hline 36 & 0.049 & 0.023 & 0.001 & 0.040 & 0.182 & 0.089 & 0.979 & 0.093 & 0.809 & 0.174 \\
\hline 37 & 0.081 & 0.896 & 1.000 & 0.064 & 1.000 & 0.216 & 0.147 & 0.594 & 0.039 & 0.022 \\
\hline 38 & 0.011 & 0.020 & 0.003 & 0.074 & 0.010 & 0.078 & 0.146 & 0.145 & 0.280 & 0.171 \\
\hline 39 & 0.039 & 0.073 & 0.007 & 0.225 & 0.111 & 0.057 & 0.262 & 0.129 & 0.090 & 0.003 \\
\hline 40 & 0.054 & 0.006 & 0.719 & 0.560 & 0.085 & 1.000 & 0.368 & 1.000 & 0.461 & 0.118 \\
\hline 41 & 0.063 & 0.099 & 0.014 & 0.258 & 0.016 & 0.184 & 0.159 & 0.285 & 0.177 & 0.063 \\
\hline
\end{tabular}

factor of elemental MSE, most elements contain at least one potential sensitive mode except elements 13, 18, 33 .

Secondly, potential sensitive modes are also chosen based on the elemental RFA by (equation (14)) in Section 3.2. The RFA of all elements for the first ten modes is calculated by the Monte Carlo method and listed in Table 3. Herein, a 5\% noise level is considered and 5000 samples are randomly generated. All RFA values that are smaller than $\gamma_{\text {RFA }}$ (prescribed as 2.00) are highlighted in bold. For each element, the mode with a highlighted value is regarded as a potential sensitive mode of the element. From Table 3, it is shown that when considering the factor of elemental RFA, most elements have at least one potential sensitive mode except element 33.

Finally, the sensitive modes for each element are determined by finding the mutual potential sensitive modes from Tables 2 and 3 and then listed in Table 4 . It is found that each element may have different sensitive modes. Most elements contain at least one sensitive mode except elements $4,13,18,33$, and all elements have no more than five sensitive modes. It also reveals that for a specific element, the mode associated with small normalized MSE is usually related to great RFA (e.g., element 40 at mode 1, 2 and element 20 at mode 3 ).

4.4. Performance of the Proposed MSECI Method Using Sensitive Modes. Since the sensitive modes of all elements have been determined in Section 4.3, how well the proposed MSECI method performs can be investigated.

It is supposed that element 9 has a $30 \%$ damage extent, with modes $1,4,6,8$, and 9 (from Table 4) as its sensitive modes. The MSECI values using modes 1, 4, 6, 8, and 9 considering both free noise and random $5 \%$ noise are then 
TABLE 3: RFA of all elements for the first ten modes based on 5000 samples.

\begin{tabular}{|c|c|c|c|c|c|c|c|c|c|c|}
\hline Element & Mode 1 & Mode 2 & Mode 3 & Mode 4 & Mode 5 & Mode 6 & Mode 7 & Mode 8 & Mode 9 & Mode 10 \\
\hline 1 & 1.182 & 1.206 & 1.146 & 1.291 & 0.984 & 1.064 & 1.100 & 0.918 & 0.904 & 0.662 \\
\hline 2 & 66.805 & 21.216 & 20.537 & 1.143 & 3.264 & 6.521 & 1.430 & 3.989 & 1.060 & 0.588 \\
\hline 3 & 694168.583 & 571.015 & 242.044 & 305.165 & 12.891 & 12.401 & 27.897 & 1.264 & 96.771 & 69.446 \\
\hline 4 & 2.959 & 18.546 & 25.874 & 3.663 & 10.777 & 3.095 & 1.019 & 2.512 & 0.919 & 0.547 \\
\hline 5 & 37.874 & 19.433 & 12.211 & 4.321 & 3.571 & 6.705 & 1.960 & 14.118 & 1.775 & 1.358 \\
\hline 6 & 33.715 & 13.896 & 18.622 & 2.869 & 5.648 & 2.237 & 2.262 & 3.542 & 1.592 & 0.596 \\
\hline 7 & 12.635 & 8.002 & 8.506 & 2.194 & 2.036 & 35.870 & 1.432 & 1.521 & 2.544 & 0.453 \\
\hline 8 & 848607.338 & 603.801 & 67.162 & 72.043 & 1.007 & 1.223 & 10.179 & 1.149 & 40.793 & 40.067 \\
\hline 9 & 1.927 & 7.783 & 13.815 & 1.667 & 16.961 & 1.743 & 3.072 & 1.449 & 1.883 & 0.500 \\
\hline 10 & 102.102 & 147.828 & 20.784 & 10.419 & 10.409 & 1.604 & 1.289 & 0.858 & 1.977 & 0.727 \\
\hline 11 & 93.771 & 37.769 & 3231.261 & 29.130 & 2.567 & 4.992 & 1.145 & 4.087 & 1.517 & 1.074 \\
\hline 12 & 7.106 & 8.897 & 7.946 & 54.439 & 1.039 & 1.427 & 1.321 & 2.791 & 2.304 & 1.236 \\
\hline 13 & 696809.642 & 201.285 & 15.709 & 22.898 & 6.881 & 8.118 & 7.643 & 0.830 & 1763.166 & 56.318 \\
\hline 14 & 2.756 & 6.079 & 56.808 & 1.064 & 1.772 & 3.393 & 1.318 & 3.976 & 3.631 & 1.517 \\
\hline 15 & 355.872 & 50.033 & 738.051 & 2.214 & 20.433 & 1.533 & 2.185 & 3.124 & 1.117 & 1.755 \\
\hline 16 & 310.576 & 58046.525 & 16.115 & 2.854 & 1.177 & 22.278 & 7.636 & 0.981 & 1.193 & 1.523 \\
\hline 17 & 5.150 & 73.595 & 6.218 & 1.975 & 1.433 & 2.542 & 1.414 & 19.800 & 1.151 & 0.537 \\
\hline 18 & 415184.882 & 16.388 & 8.815 & 56.444 & 1.754 & 2.141 & 16651.839 & 2.556 & 1.077 & 14.424 \\
\hline 19 & 3.790 & 6.094 & 17.193 & 1.086 & 1.834 & 9.851 & 3.881 & 10.489 & 1.178 & 3.431 \\
\hline 20 & 2238.547 & 11.099 & 39.441 & 3.977 & 1.473 & 5.960 & 1.825 & 13.747 & 1.672 & 0.997 \\
\hline 21 & 5591.630 & 31.153 & 7.413 & 11.384 & 11.340 & 1.227 & 1.490 & 14.934 & 1.100 & 0.739 \\
\hline 22 & 3.809 & 16.723 & 25.062 & 1.317 & 2.317 & 14.994 & 2.921 & 2.737 & 1.502 & 1.083 \\
\hline 23 & 390811.762 & 29.762 & 24.784 & 21.538 & 11.750 & 46.752 & 7.612 & 1.259 & 30.240 & 304265.773 \\
\hline 24 & 6.618 & 28.130 & 5.991 & 2.215 & 5.521 & 2.097 & 1.151 & 2.809 & 1.633 & 0.658 \\
\hline 25 & 7104.093 & 7.025 & 220.131 & 7.568 & 2.355 & 8.558 & 1.488 & 12.037 & 1.265 & 0.888 \\
\hline 26 & 3912.675 & 20.857 & 7.225 & 3.838 & 6.857 & 2.808 & 1.282 & 1.691 & 1.973 & 1.427 \\
\hline 27 & 3.194 & 6.362 & 717.301 & 31.821 & 4.172 & 5.672 & 1.136 & 2.206 & 3.738 & 1.182 \\
\hline 28 & 105034.178 & 60.258 & 19.701 & 46.713 & 51.182 & 8.911 & 11.000 & 0.956 & 1026.552 & 7.741 \\
\hline 29 & 8.429 & 7.918 & 3.324 & 2.556 & 12.571 & 8.238 & 1.046 & 1.580 & 2.061 & 0.780 \\
\hline 30 & 376.689 & 9.473 & 8.069 & 3.678 & 14.814 & 2.999 & 1.673 & 0.613 & 1.406 & 1.548 \\
\hline 31 & 342.848 & 242.076 & 13.517 & 2.040 & 3.910 & 3.581 & 10.612 & 1.404 & 1.122 & 1.867 \\
\hline 32 & 1.694 & 3.098 & 10.337 & 2.285 & 10.063 & 18.386 & 3.733 & 3.142 & 1.219 & 0.574 \\
\hline 33 & 41569.701 & 40.179 & 9.353 & 74.833 & 8.983 & 9.808 & 14.658 & 151.314 & 11.205 & 18.625 \\
\hline 34 & 14.170 & 6.034 & 1.922 & 1.420 & 11.979 & 3.376 & 1.513 & 4.843 & 2.013 & 0.519 \\
\hline 35 & 98.570 & 13.704 & 4.441 & 470.300 & 2.899 & 84.483 & 1.877 & 7.033 & 1.290 & 1.480 \\
\hline 36 & 84.501 & 28.524 & 184.576 & 11.396 & 3.118 & 3.147 & 0.989 & 4.237 & 1.668 & 0.490 \\
\hline 37 & 1.360 & 1.415 & 3.392 & 1.744 & 2.204 & 5.168 & 2.560 & 5.972 & 15.776 & 2.076 \\
\hline 38 & 1.134 & 1.454 & 1.224 & 1.331 & 1.107 & 1.244 & 0.964 & 0.952 & 1.165 & 0.708 \\
\hline 39 & 69.443 & 12.231 & 4.426 & 4.004 & 4.905 & 3.047 & 1.229 & 5.392 & 1.139 & 0.630 \\
\hline 40 & 25.640 & 48.586 & 1.278 & 3.450 & 2.055 & 1.946 & 1.869 & 5.788 & 3.575 & 1.224 \\
\hline 41 & 38.847 & 8.614 & 11.455 & 4.789 & 11.079 & 2.499 & 2.335 & 2.968 & 1.706 & 0.911 \\
\hline
\end{tabular}

TABLE 4: Determined sensitive modes of all elements.

\begin{tabular}{|c|c|c|c|c|c|c|c|}
\hline Element & Modes & Element & Modes & Element & Modes & Element & Modes \\
\hline 1 & $7,9,10$ & 12 & 6,7 & 23 & 8 & 34 & 3,4 \\
\hline 2 & $4,7,9$ & 13 & - & 24 & 7,9 & 35 & $7,9,10$ \\
\hline 3 & 8 & 14 & $4,5,7$ & 25 & $7,9,10$ & 36 & $7,9,10$ \\
\hline 4 & - & 15 & $6,9,10$ & 26 & $7,8,9,10$ & 37 & 2 \\
\hline 5 & 7,9 & 16 & $5,8,9,10$ & 27 & 7 & 38 & $7,8,9,10$ \\
\hline 6 & 9 & 17 & $4,7,9$ & 28 & 8 & 39 & 7,9 \\
\hline 7 & 8 & 18 & - & 29 & 7,8 & 40 & $3,6,7,10$ \\
\hline 8 & 8 & 19 & $4,5,9$ & 30 & $7,8,9,10$ & 41 & 9 \\
\hline 9 & $1,4,6,8,9$ & 20 & $5,7,9,10$ & 31 & 9,10 & & \\
\hline 10 & $6,7,8,9,10$ & 21 & $6,7,9,10$ & 32 & 1 & & \\
\hline 11 & $7,9,10$ & 22 & 4,9 & 33 & - & & \\
\hline
\end{tabular}

calculated and drawn in Figure 8. Comparing the results shown in Figures 6-8, it is discovered that the MSECI method using sensitive modes is obviously more indicative than just using the first several modes together. It is noticed that modes 4, 8 (from Tables 2 and 3) have relatively great MSE values and small RFA values, meaning that they are more sensitive than modes $1,6,9$. Thus, only modes 4 and 8 are selected to calculate the MSECI values again, with the 


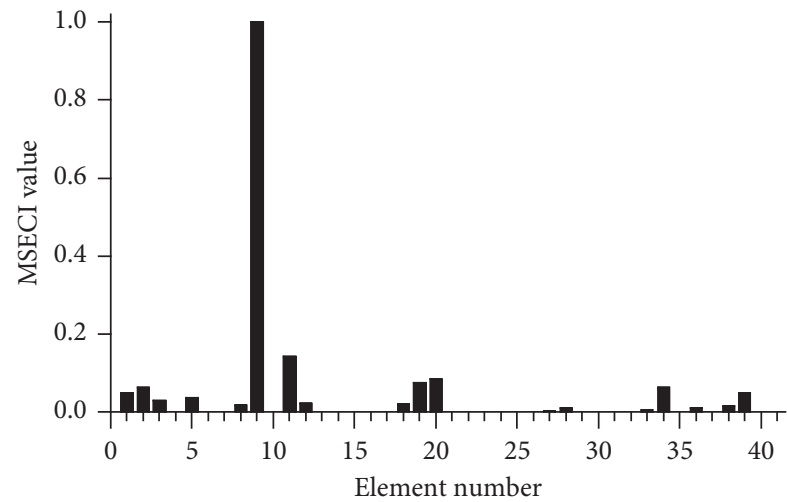

(a)

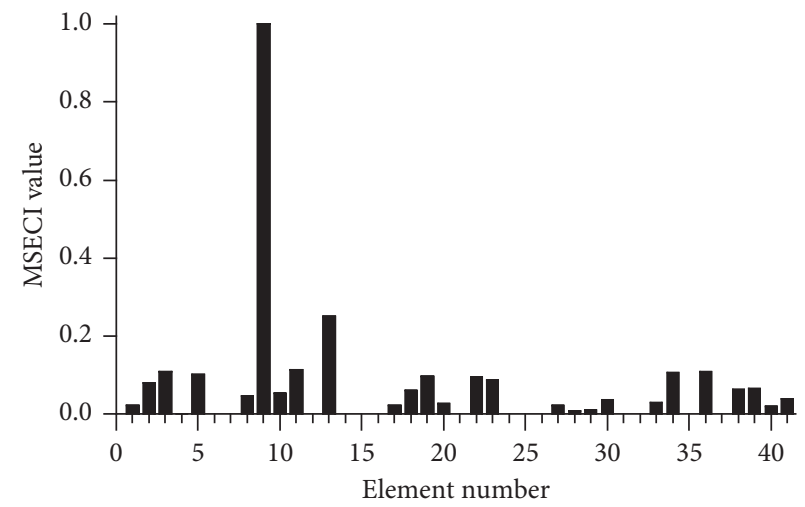

(b)

FIgURE 8: Values of the proposed MSECI using modes 1, 4, 6, 8, 9 (damaged element 9). (a) Without noise. (b) With 5\% noise.

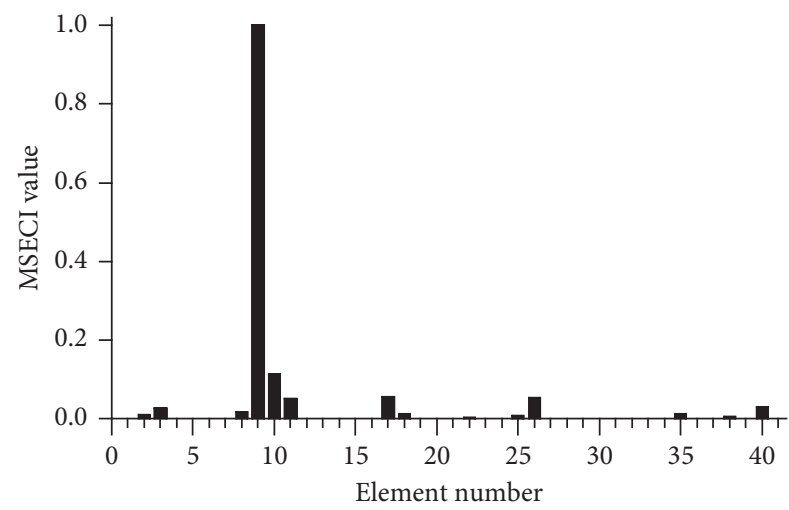

(a)

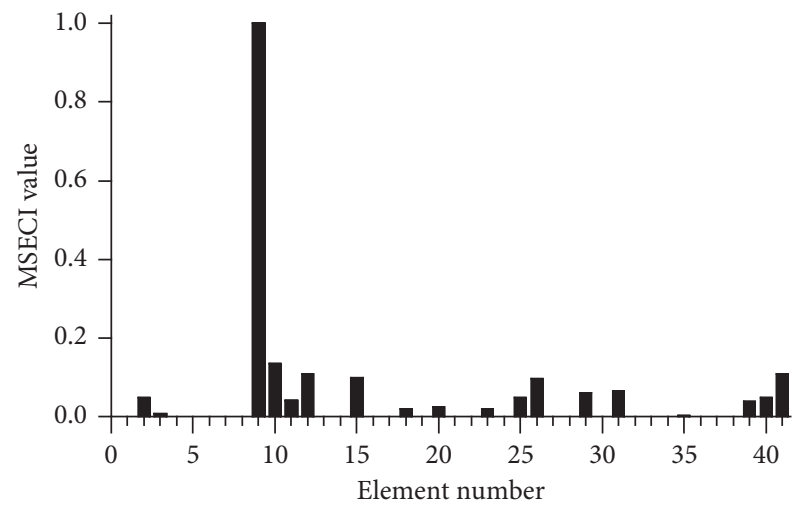

(b)

Figure 9: Values of the proposed MSECI using modes 4, 8 (damaged element 9). (a) Without noise. (b) With 5\% noise.

results depicted in Figure 9. Comparing Figures 8 and 9, using modes 4 and 8 only can further improve the robustness of the MSECI method.

It is concluded from the above analysis that insensitive modes do harm to the robustness of the proposed MSECI method (Figures 6-7), but merely using sensitive modes can make it perform more reliably when considering a relatively high noise level (Figures 8-9).

\subsection{In-Turn Damage-Checking by the MSECI Method Using} Sensitive Modes. Because the damaged element is unknown in a real structure and the sensitive modes are usually different for each element, it would be better to check the damage state of each element in turn if using the proposed method. Take the damage scenario in which both elements 11 and 37 are damaged by $30 \%$ for example to illustrate the in-turn damage-checking process.

Although each element should be checked one by one, only elements $8,11,14,19,37$, and 38 are checked herein due to space limitation. If it is an element's turn to check its damage state, only the sensitive modes given in Table 4 of the element are used to calculate the MSECI values. For each element's turn, if the element's MSECI value exceeds other elements' values, the element can be judged as a damaged element. Otherwise, it is treated as being undamaged.

The calculated results considering $5 \%$ random noise are drawn in Figure 10. From Figure 10(a), because the MSECI value of element 8 is very small, it is judged as being undamaged. Similarly, element 14 in Figure 10(c), element 19 in Figure $10(\mathrm{~d})$, and element 38 in Figure 10(f) can be easily identified as intact elements. On the contrary, from Figure 9(b), the MSECI value of element 11 significantly outweighs other elements' values, so it is successfully determined as a damaged element. Analogously, it is judged from Figure 10(e) that element 37 is in damaged state. In this in-turn damage-checking way, the damage state of each element can be reliably determined by the proposed MSECI method.

If the first several modes are taken into account together, the MSECI values considering both free noise and 5\% random noise are shown in Figure 11. It is discovered from Figure 11(b) that the damaged element 37 can be clearly determined, but the damaged element 11 is greatly disturbed by several other undamaged elements. Hence, in the traditional way of using the first several modes together, the damaged elements can hardly be located reliably.

By far, it is roughly inferred that the proposed MSECI method can provide reliable damage localization results if 


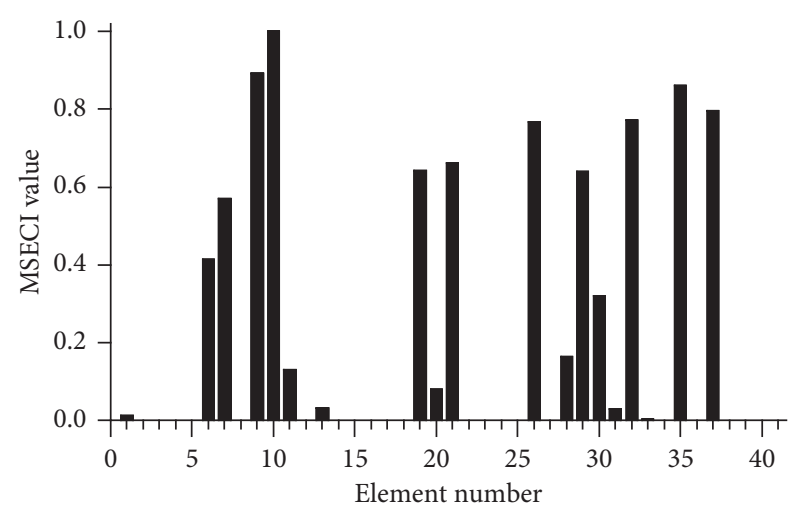

(a)

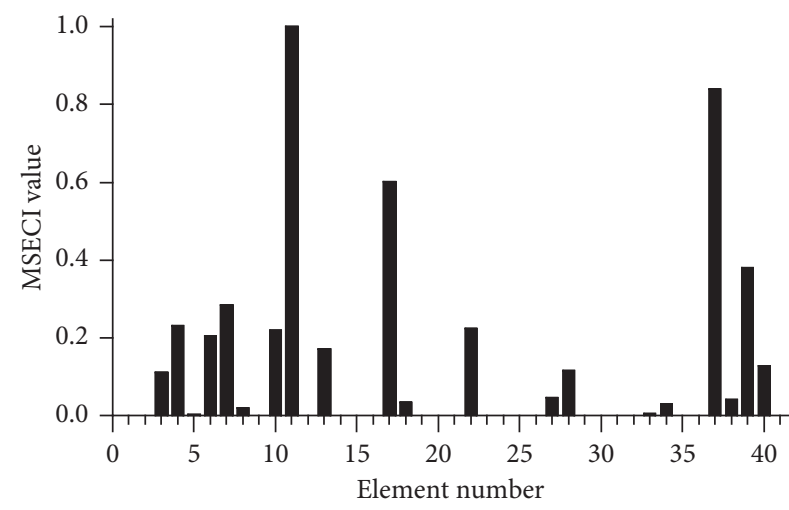

(c)

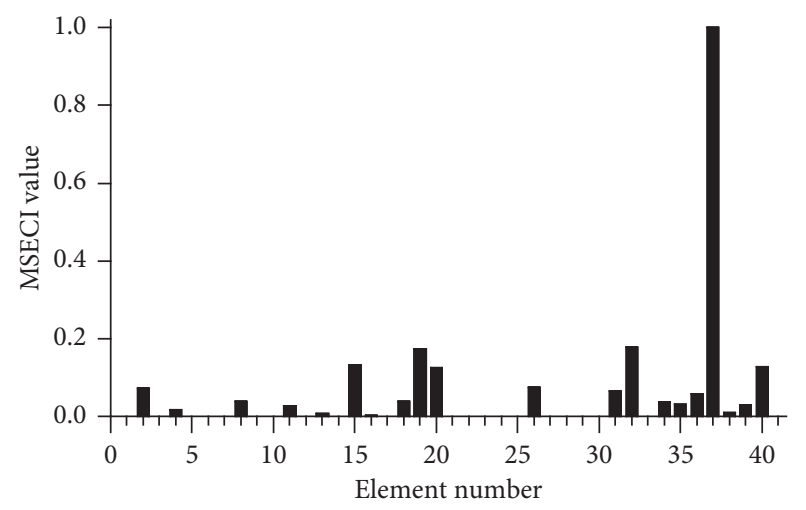

(e)

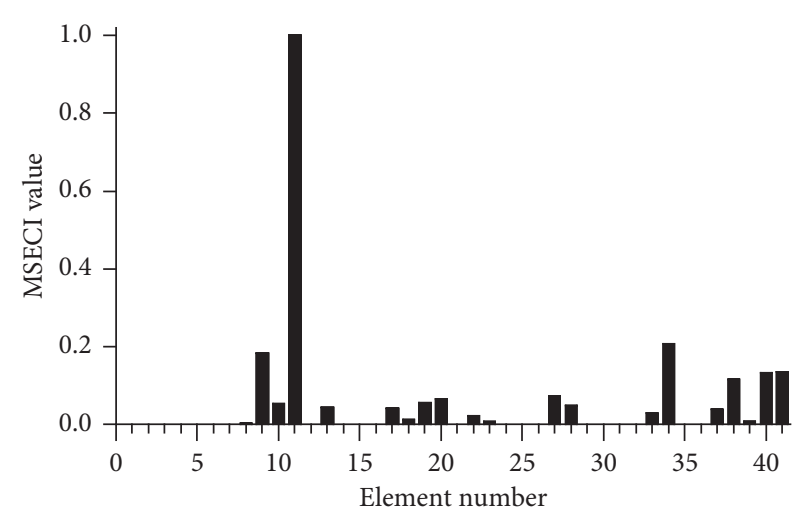

(b)

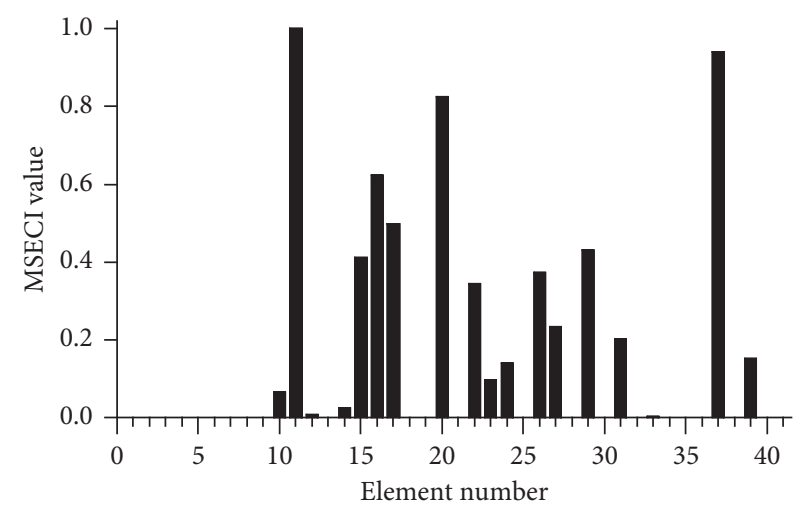

(d)

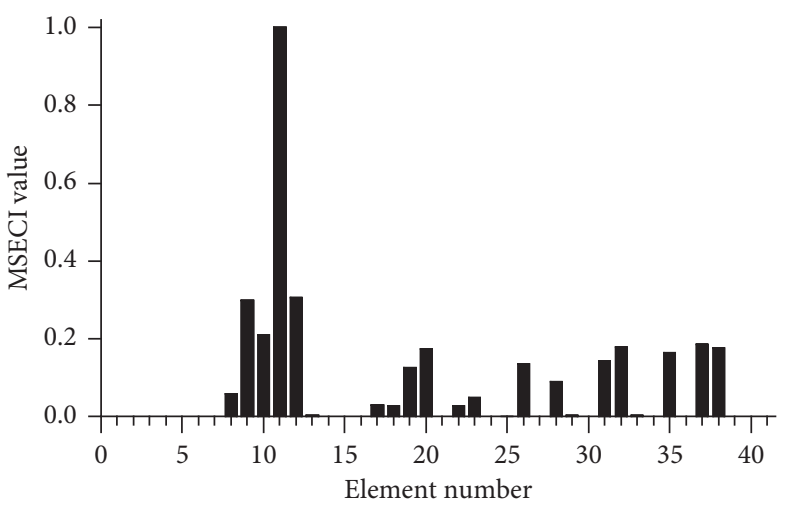

(f)

Figure 10: Values of the proposed MSECI using sensitive modes for in-turn checking (damaged element 11, 37, and 5\% noise). (a) Checking element 8, using modes 8. (b) Checking element 11, using modes 7, 9, and 10. (c) Checking element 14, using modes 4, 5, and 7. (d) Checking element 19, using modes 4, 5, 9. (e) Checking element 37, using mode 2. (f) Checking element 38, using modes 7, 8, 9, and 10.

using sensitive modes only and checking each element's damage state in turn.

\subsection{Statistic Performance of the Proposed MSECI Method.} Due to the randomness of identification uncertainties, the statistic performance of the proposed method with an in-turn damage-checking process mentioned in Section 4.5 is worth investigating. If it is element $j$ 's turn, the ratio of the element's MSECI value to the largest MSECI value of other elements, which is denoted as $R_{j}$, should be calculated by using its sensitive modes only shown in Table 4 . Herein, the first eight modes are utilized together for an element containing no sensitive modes. Besides, a 5\% noise level and 5000 samples are considered. If $R_{j}$ of any sample is greater than 1.00 , element $j$ has the largest MSECI value and can be judged as being damaged. The ratio of number of times that element $j$ is judged as a damaged element to the total number of samples is defined as the detection probability of element $j$. Obviously, great detection probabilities of damaged elements and small detection probabilities of intact elements statistically mean good performance of the proposed MSECI method.

Damage scenario 1 (damaged elements 11,37) and damage scenario 2 (damaged elements 14, 18, 35) are considered to 


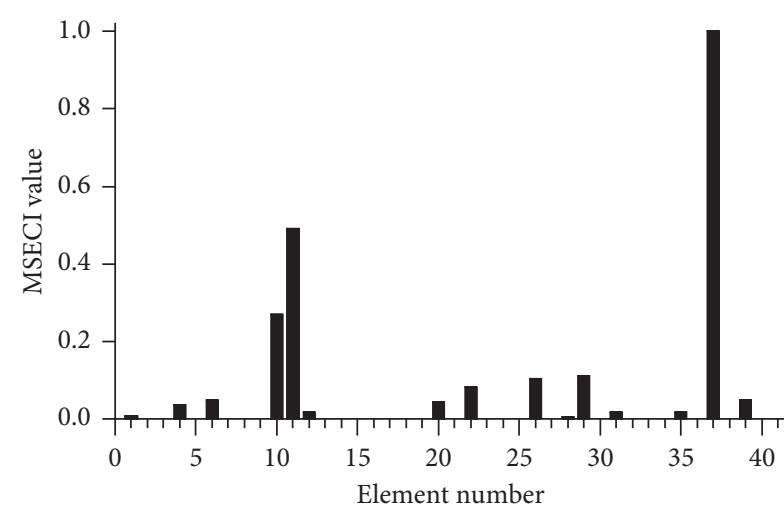

(a)

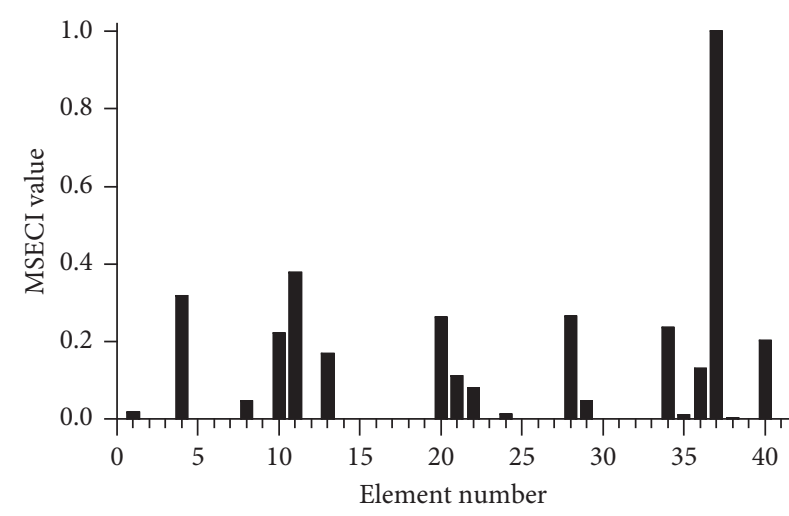

(b)

Figure 11: Values of the proposed MSECI using modes 1-8 (damaged element 11, 37). (a) Without noise. (b) With 5\% noise.

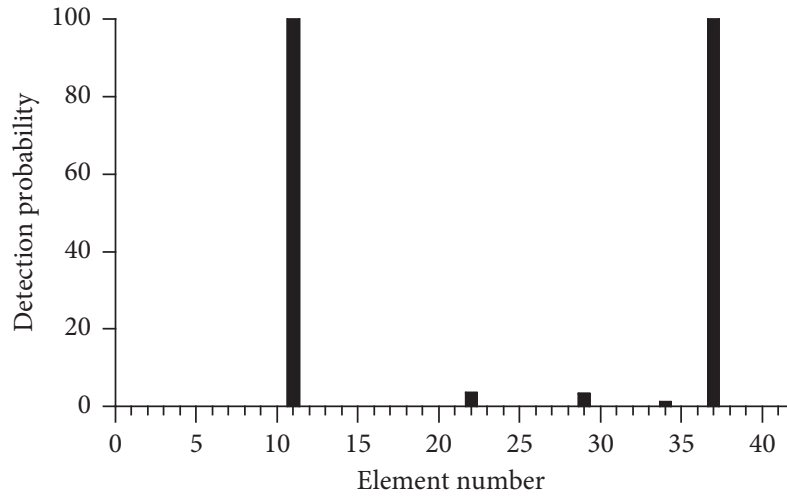

(a)

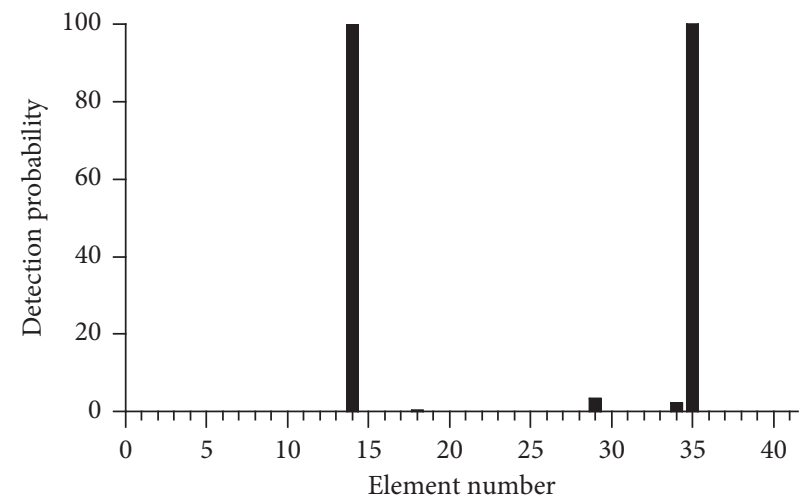

(b)

FIGURE 12: Detection probabilities calculated by the proposed MSECI method with an in-turn damage-checking process (5\% noise and 5000 samples). (a) Scenario 1 (damaged element 11 and 37). (b) Scenario 2 (damaged element 14, 18, and 35).

demonstrate the detection probabilities of all elements, as shown in Figure 12. In the two scenarios, all damaged elements are damaged by $30 \%$. For scenario 1 , it reveals from Figure 12 (a) that both damaged elements 11 and 37 have a 100\% detection probability, while most other intact elements' detection probabilities equal to $0 \%$, with elements 22,29 , and 34 's detection probabilities between $1.14 \%$ and $3.58 \%$. Besides, though elements $4,13,18,33$ contain no sensitive modes, their undamaged states can be correctly judged in each sample for each sample. For scenario 2, it exhibits from Figure 12(b) that the detection probabilities of damaged elements 14, 18, and 35 are 99.96\%, $0.2 \%$ and $100 \%$, respectively. Meanwhile, most intact elements have a $0 \%$ detection probability, except two intact elements 29 , 34 , which have a detection probability of $3.34 \%$ and $2.2 \%$, respectively. It is noted that for elements having no sensitive modes, damaged element 18 is misjudged as being undamaged nearly for each sample, but intact elements 4,13 , and 33 are always correctly detected as being undamaged.

It is concluded from Figure 12 that by using the proposed method with an in-turn damage-checking process, the damage states of most elements which contain sensitive modes can be reliably judged, but it is nearly impossible to judge whether the few elements having no sensitive modes are damaged or not.

\section{Conclusion}

MSE-based methods traditionally utilize the first several modes together to calculate the damage location indicators. Because of the participation of insensitive modes, these methods are not robust enough and sometimes even lose effectiveness. Thus, the MSECI method that establishes a novel damage location indicator and uses properly selected sensitive modes is proposed in this paper. Both the elemental MSE of the intact structure and the capability of each element to resist noise are considered to distinguish sensitive modes from the first several modes. Since each element usually has different sensitive modes, an in-turn damage-checking process is required to finish the damage localization.

The numerical results show that the proposed MSECI method performs better than the classic MSECR method even when considering the first several modes together. It is found that for the proposed method, elements normally contain different number of sensitive modes. MSECI values calculated by merely using sensitive modes are like a magnifying glass that can easily find local damage, so each element's damage state can be reliably checked in turn, except a few elements that have no sensitive modes. It is speculated that whether an element has sensitive modes or 
not depends on the configuration of the structure, including geometry, topology, etc. Generally speaking, the proposed method can greatly improve the robustness of damage localization of the planar truss. It is worth mentioning that the stiffness matrix of a real intact structure may be different from that of the finite element model. Thus, model updating techniques should be introduced to update the stiffness matrix to ensure the accuracy of proposed MSECI values.

The work indicates that the sensitivity of modes to elemental damage varies, and the performance of the proposed method for other structures needs further investigation.

\section{Data Availability}

The data used to support the findings of this study are available from the corresponding author upon request.

\section{Conflicts of Interest}

The authors declare that they have no conflicts of interest.

\section{Acknowledgments}

This work was supported by the National Natural Science Foundation of China (Grant number 51868026), the Science and Technology Project of Jiangxi Educational Committee (Grant number GJJ170529), and the Doctoral Scientific Research Foundation of Jiangxi University of Science and Technology.

\section{References}

[1] O. Hattab, M. Chaari, M. A. Franchek, and T. Wassar, "An adaptive modeling approach to structural health monitoring of multistory buildings," Journal of Sound and Vibration, vol. 440, pp. 239-255, 2019.

[2] C.-M. Chang, T.-K. Lin, and C.-W. Chang, "Applications of neural network models for structural health monitoring based on derived modal properties," Measurement, vol. 129, pp. 457-470, 2018.

[3] Y. Y. Li, "Hypersensitivity of strain-based indicators for structural damage identification: a review," Mechanical Systems and Signal Processing, vol. 24, no. 3, pp. 653-664, 2010.

[4] A. H. Hadjian Shahri and A. K. Ghorbani-Tanha, "Damage detection via closed-form sensitivity matrix of modal kinetic energy change ratio," Journal of Sound and Vibration, vol. 401, pp. 268-281, 2017.

[5] S. Talaei, A. Beitollahi, S. Moshirabadi, and M. Fallahian, "Vibration-based structural damage detection using Twin Gaussian Process (TGP)," Structures, vol. 16, pp. 10-19, 2018.

[6] J. Ciambella, A. Pau, and F. Vestroni, "Modal curvature-based damage localization in weakly damaged continuous beams," Mechanical Systems and Signal Processing, vol. 121, pp. 171$182,2019$.

[7] Y. Q. Ni, H. F. Zhou, K. C. Chan, and J. M. Ko, "Modal flexibility analysis of cable-stayed Ting Kau bridge for damage identification," Computer-Aided Civil and Infrastructure Engineering, vol. 23, no. 3, pp. 223-236, 2008.

[8] M. Nobahari, M. R. Ghasemi, and N. Shabakhty, "A fast and robust method for damage detection of truss structures," Applied Mathematical Modelling, vol. 68, pp. 368-382, 2019.
[9] Z. Y. Shi, S. S. Law, and L. M. Zhang, "Structural damage localization from modal strain energy change," Journal of Sound and Vibration, vol. 218, no. 5, pp. 825-844, 1998.

[10] W.-J. Yan, W.-X. Ren, and T.-L. Huang, "Statistic structural damage detection based on the closed-form of element modal strain energy sensitivity," Mechanical Systems and Signal Processing, vol. 28, pp. 183-194, 2012.

[11] S. M. Seyedpoor, "A two stage method for structural damage detection using a modal strain energy based index and particle swarm optimization," International Journal of Non-linear Mechanics, vol. 47, no. 1, pp. 1-8, 2012.

[12] T. Vo-Duy, V. Ho-Huu, H. Dang-Trung, and T. Nguyen-Thoi, "A two-step approach for damage detection in laminated composite structures using modal strain energy method and an improved differential evolution algorithm," Composite Structures, vol. 147, pp. 42-53, 2016.

[13] Z. X. Tan, D. P. Thambiratnam, T. H. T. Chan, and H. A. Razak, "Detecting damage in steel beams using modal strain energy based damage index and Artificial Neural Network," Engineering Failure Analysis, vol. 79, pp. 253-262, 2017.

[14] G. Liu, Y. Zhai, D. Leng, X. Tian, and W. Mu, "Research on structural damage detection of offshore platforms based on grouping modal strain energy," Ocean Engineering, vol. 140, pp. 43-49, 2017.

[15] J. T. Kim and N. Stubbs, "Improved damage identification method based on modal information," Journal of Sound and Vibration, vol. 252, no. 2, pp. 223-238, 2002.

[16] K. Nguyen, T. H. T. Chan, and D. P. Thambiratnam, "Structural damage identification based on change in geometric modal strain energy-eigenvalue ratio," Smart Materials and Structures, vol. 25, no. 7, Article ID 075032, 2016.

[17] S. W. Doebling, F. M. Hemez, L. D. Peterson, and C. Farhat, "Improved damage location accuracy using strain energybased mode selection criteria," AIAA Journal, vol. 35, pp. 693-699, 1997. 


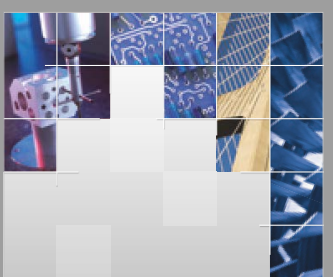

\section{Enfincering}
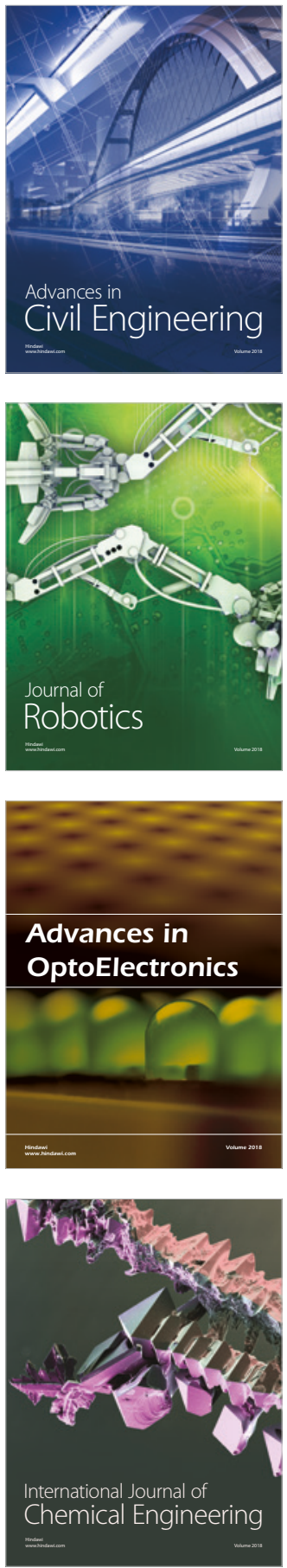

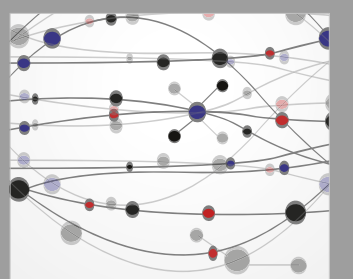

\section{Rotating \\ Machinery}

The Scientific World Journal

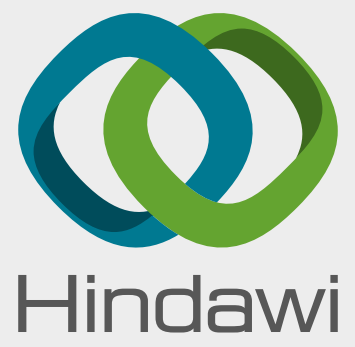

Submit your manuscripts at

www.hindawi.com
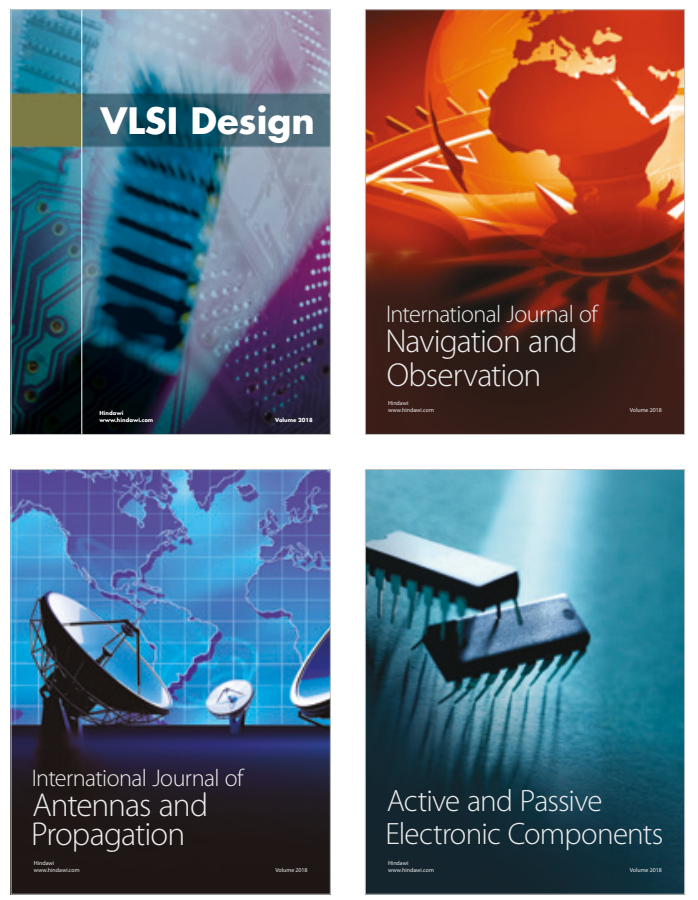
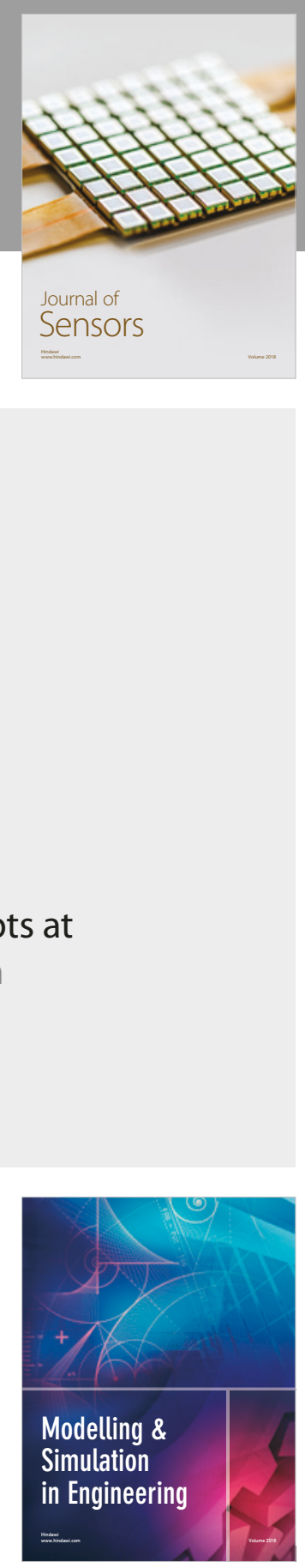

\section{Advances \\ Multimedia}
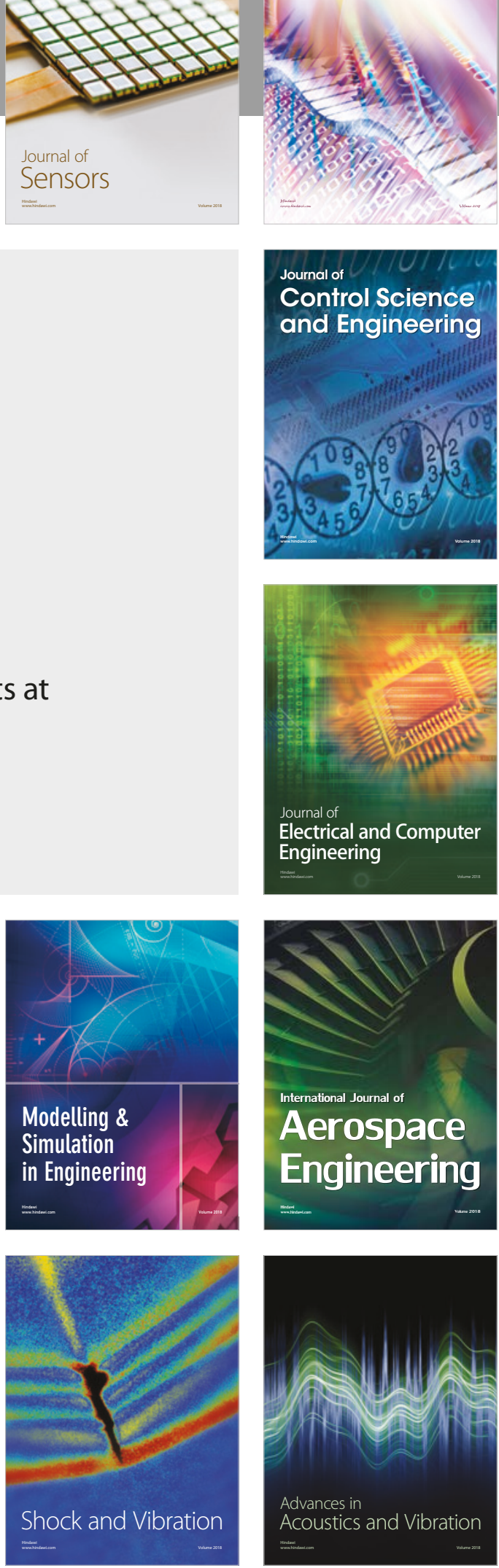